JOURNAL OF

SYMPLECTIC GEOMETRY

Volume 9, Number 1, 33-44, 2011

\title{
LEGENDRIAN CONTACT HOMOLOGY AND NONDESTABILIZABILITY
}

\author{
Clayton Shonkwiler and David Shea Vela-Vick
}

We provide the first example of a Legendrian knot with nonvanishing contact homology whose Thurston-Bennequin invariant is not maximal.

\section{Introduction}

Since it was proposed by Etnyre [Etn99] and first implemented by Etnyre and Honda [EH01], the most common strategy for classifying Legendrian knots in a given knot type $K$ has been to approach the problem in two steps. First, find all Legendrian representatives of $K$ with maximal ThurstonBennequin invariant, then attempt to show that all other Legendrian representatives of $K$ can be destabilized to one of these maximal examples.

This method has proven quite effective, but, as observed by Etnyre and Honda [EH05], not all nondestabilizable Legendrian knots have maximal Thurston-Bennequin invariant. Thus, one needs a means for determining which Legendrian knots are nondestabilizable.

A candidate for identifying nondestabilizable Legendrian knots is Legendrian contact homology, which has been one of the most powerful nonclassical invariants of Legendrian knots since it was defined by Chekanov [Che02] and Eliashberg [Eli98]. This invariant, which takes the form of a differential graded algebra $(\mathcal{A}, \partial)$ and is a specialized variant of symplectic field theory [EGH00], vanishes for stabilized Legendrian knots and is nonvanishing for every nondestabilizable Legendrian knot for which it has been computed. All such examples to date have had maximal Thurston-Bennequin invariant, but in Theorem 1.1 we show that the Legendrian contact homology is nonvanishing for a certain nondestabilizable Legendrian knot with nonmaximal Thurston-Bennequin invariant.

We do this by showing that a related invariant, the characteristic algebra, is nontrivial. The characteristic algebra was defined by $\mathrm{Ng}[\mathbf{N g 0 1}]$ as 
$C(L):=\mathcal{A}_{F} /\langle\operatorname{im} \partial\rangle$, where $F$ is a front diagram for $L, \mathcal{A}_{F}$ is the free, noncommutative, unital $\mathbb{Z} / 2$-algebra generated by the crossings and right cusps of $F$, and $\langle\operatorname{im} \partial\rangle \subset \mathcal{A}_{F}$ is the two-sided ideal generated by the image of the contact homology differential. If $L$ and $L^{\prime}$ are Legendrian isotopic, then the characteristic algebras $C(L)$ and $C\left(L^{\prime}\right)$ become tamely isomorphic after adding some (possibly different) number of generators to each.

$\mathrm{Ng}$ conjectured that the characteristic algebra of a nondestabilizable Legendrian knot is nonvanishing [Ng01, Conjecture 6.4.1], which would imply that the Legendrian contact homology for such knots is also nonvanishing (see Proposition 3.1). We give some evidence for Ng's conjecture by providing the first example of a Legendrian knot with nonvanishing characteristic algebra which does not have maximal Thurston-Bennequin invariant.

Theorem 1.1. The contact homology and characteristic algebra of Chongchitmate and $\mathrm{Ng}$ 's nondestabilizable Legendrian $\mathrm{m}\left(10_{161}\right)$ are nonvanishing.

Remark 1.1. A similar argument to that given in the proof of Theorem 1.1 shows that the contact homology and characteristic algebra of Chongchitmate and Ng's nondestabilizable Legendrian $m\left(10_{145}\right)$ are also nonvanishing.

Remark 1.2. There is a lift of the contact homology and characteristic algebra to $\mathbb{Z}\left[t, t^{-1}\right]$ (see [ENS02]). Nonvanishing over $\mathbb{Z} / 2$ implies nonvanishing in the more general $\mathbb{Z}\left[t, t^{-1}\right]$ setting.

The general situation is still far from clear, however, as we also provide some evidence against Ng's conjecture. Chongchitmate and $\mathrm{Ng}$ exhibited a Legendrian $m\left(10_{139}\right)$ which does not have maximal Thurston-Bennequin invariant and which they conjectured, based on computational evidence, is nondestabilizable and sits atop its own peak in the $t b-r$ mountain range. In Section 4 we prove:

Proposition 1.1. The contact homology and characteristic algebra of Chongchitmate and $N g$ 's Legendrian $m\left(1_{139}\right)$ vanish identically over $\mathbb{Z}\left[t, t^{-1}\right]$.

Assuming this knot is actually nondestabilizable, this would provide the first example of a nondestabilizable Legendrian knot with vanishing characteristic algebra or contact homology. This suggests the following:

Conjecture 1.1. There exist nondestabilizable Legendrian knots with vanishing contact homology.

For background information on Legendrian knots and Legendrian contact homology, we refer the reader to Etnyre's survey [Etn05]. 


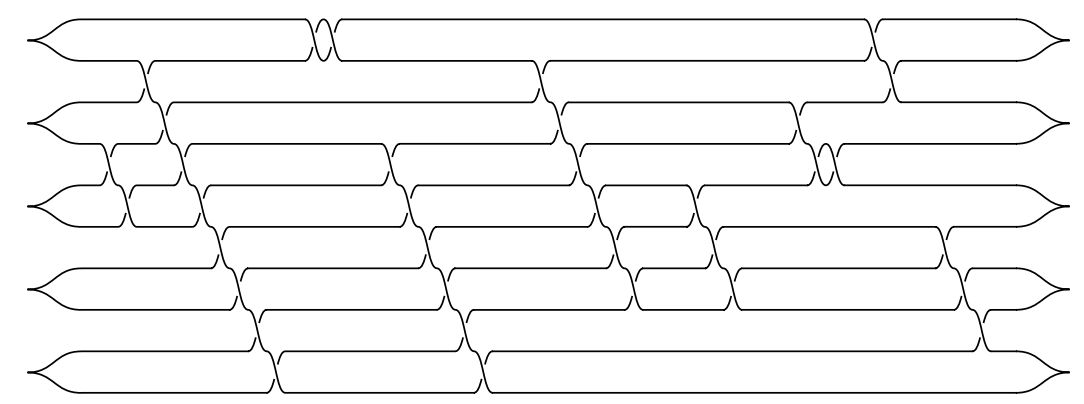

Figure 1. Chongchitmate and Ng's nondestabilizable $m\left(10_{161}\right)$.

\section{The $m\left(10_{161}\right)$}

As mentioned in the Introduction, Etnyre and Honda [EH05] presented the first example of a nondestabilizable Legendrian knot whose ThurstonBennequin invariant is nonmaximal for its knot type. This example is a Legendrian (2,3)-cable of the (2,3)-torus knot.

Recently, Chongchitmate and Ng produced a conjectural atlas [CN09] for low-crossing Legendrian knots. Included in this atlas are several new examples of nondestabilizable Legendrian knots whose Thurston-Bennequin invariants are not maximal. In particular, Chongchitmate and $\mathrm{Ng}$ give examples of nondestabilizable Legendrian $m\left(1_{161}\right)$ and $m\left(10_{145}\right)$ whose Thurston-Bennequin invariants are nonmaximal ( $m$ here stands for "mirror").

For the purposes of computing the contact homology differential for a Legendrian knot, it is useful to have it presented as the plat closure of a positive braid. Using Chongchitmate and Ng's original presentation, it is not difficult to derive the plat diagram for the $m\left(1_{161}\right)$ appearing in Figure 1.

The braid word defining the plat diagram in Figure 1 is

$4,5,2,3,4,5,6,7,8,9,1,1,4,5,6,7,8,9,2,3,4,5,6,7,5,6,7,3,4,4,1,2,6,7,8$.

In Figure 1 there are a total of 35 crossings and five right cusps. The crossings are labeled $x_{1}$ through $x_{35}$ from left to right and the right cusps are labeled $x_{36}$ through $x_{40}$ from top to bottom. Therefore, for this front diagram for the $m\left(10_{161}\right), \mathcal{A}_{m\left(10_{161}\right)}$ is equal to $\mathbb{Z} / 2\left\langle x_{1}, \ldots, x_{40}\right\rangle$, the free unital $\mathbb{Z} / 2$-algebra of rank 40 generated by $x_{1}, \ldots, x_{40}$. The full boundary map is given in Appendix A.

\section{The proof of Theorem 1.1}

We begin with a straightforward observation relating (non)vanishing properties of the characteristic algebra to contact homology. 
Proposition 3.1. Let $L$ be a Legendrian knot in the standard contact 3-sphere. If the characteristic algebra of $L$ is nontrivial, then so is its contact homology.

Proof. Suppose that the contact homology

$$
\mathrm{CH}(L)=\frac{\operatorname{ker} \partial}{\operatorname{im} \partial}
$$

of $L$ is trivial. Then, since $\partial(1)=0$, it must be the case that im $\partial$ contains the unit element 1 . This implies that 1 must also be contained in the twosided ideal $\langle\operatorname{im} \partial\rangle$ generated by the image of the boundary map inside the full algebra $\mathcal{A}_{L}$. Therefore, the characteristic algebra of $L$ also vanishes, completing the proof of Proposition 3.1.

By Proposition 3.1, Theorem 1.1 will follow if we can show that the characteristic algebra of the Legendrian $m\left(10_{161}\right)$ depicted in Figure 1 is nontrivial.

The characteristic algebra $C\left(m\left(10_{161}\right)\right)=\mathcal{A}_{m\left(10_{161}\right)} /\langle\operatorname{im} \partial\rangle$ is

$$
C\left(m\left(10_{161}\right)\right)=\mathbb{Z} / 2\left\langle x_{1}, \ldots, x_{40}\right\rangle /\left\langle\partial x_{1}, \ldots, \partial x_{40}\right\rangle .
$$

From the differential we have that

$$
\begin{aligned}
\partial x_{2} & =x_{1}, \quad \partial x_{6}=x_{3}, \quad \partial x_{5}=x_{3} x_{2}+x_{4}, \quad \partial x_{8}=x_{7} \\
\partial x_{10} & =x_{9}, \quad \partial x_{15}=x_{14}, \quad \partial x_{17}=x_{16}, \quad \text { and } \quad \partial x_{26}=x_{25},
\end{aligned}
$$

so, in $C\left(m\left(10_{161}\right)\right)$,

$$
x_{1}=x_{3}=x_{4}=x_{7}=x_{9}=x_{14}=x_{16}=x_{25}=0 .
$$

To show that $C\left(m\left(10_{161}\right)\right) \neq 0$ we will actually show that a quotient, $\bar{C}=C\left(m\left(10_{161}\right)\right) / \mathcal{J}$, is nontrivial.

Define $\mathcal{J}$ as the two-sided ideal generated by the elements

$$
\begin{gathered}
x_{5}, x_{6}, x_{8}, x_{10}, x_{15}, x_{17}, x_{18}, x_{19}, x_{20}, x_{21}, x_{22}, x_{23}, x_{24}, x_{26}, x_{31}, x_{32}, x_{35}, x_{36}, \\
x_{37}, x_{38}, x_{39}, x_{40}, x_{30}+1, x_{34}+1, x_{27} x_{2}+1, x_{11} x_{2}, x_{28}+x_{2}, x_{11}+x_{33}
\end{gathered}
$$

and let

$$
\bar{C}:=C\left(m\left(10_{161}\right)\right) / \mathcal{J} .
$$

Using (3.1) and the relations of $\mathcal{J}$, the defining relations of $C\left(m\left(10_{161}\right)\right)$ (i.e., the boundary maps in Appendix A) can be simplified as

$$
\begin{array}{r}
x_{2} x_{13}+x_{12} x_{11}=1 \\
x_{11} x_{12}+x_{27} x_{12}=0
\end{array}
$$




$$
\begin{aligned}
x_{13} x_{2} & =1 \\
x_{11}\left(x_{29}+1\right) & =1 \\
\left(x_{29}+1\right) x_{11}+x_{2} x_{27} & =1 \\
x_{27} x_{12} & =1 \\
x_{27} x_{2} & =1 \\
x_{11} x_{2} & =0 .
\end{aligned}
$$

Therefore, $\bar{C}$ is isomorphic to $\mathbb{Z} / 2\left\langle x_{2}, x_{11}, x_{12}, x_{13}, x_{27}, x_{29}\right\rangle$ modulo the relations in (3.2).

Lemma 3.1. The algebra $\bar{C}$ is isomorphic to the algebra

$$
\mathbb{Z} / 2\langle a, b, c, d\rangle /\langle a c+d b=1, b a=0, b d=1, c a=1, c d=0\rangle .
$$

Proof. Define the map

$$
\begin{aligned}
x_{12} & \mapsto a \\
x_{13} & \mapsto b \\
x_{27} & \mapsto c \\
x_{29}+1 & \mapsto d \\
x_{2} & \mapsto e \\
x_{11} & \mapsto f .
\end{aligned}
$$

Under this map, the relations in (3.2) become

$$
\begin{aligned}
e b+a f & =1 \\
f a+c a & =0 \\
b e & =1 \\
f d & =1 \\
d f+e c & =1 \\
c a & =1 \\
c e & =1 \\
f e & =0,
\end{aligned}
$$

so $\bar{C}$ is isomorphic to $\mathbb{Z} / 2\langle a, b, c, d, e, f\rangle$ modulo these relations.

Note that, by adding (3.4) to (3.8), the above relations imply

$$
f a=1 \text {. }
$$

Now, we claim that the relations in (3.3)-(3.10) are equivalent to the relations

$$
c a=1
$$




$$
\begin{aligned}
b+c+f & =0 \\
b a & =0 \\
a+d+e & =0 \\
c d & =0 \\
b d & =1 \\
a c+d b & =1 .
\end{aligned}
$$

The relations (3.3)-(3.10) imply the relations (3.12)-(3.18) as follows:

- The relation (3.12) already appears as (3.8).

- Multiply (3.3) on the left by $c$ and simplify using (3.8) and (3.9) to get (3.13).

- Multiply (3.13) on the right by $a$ and simplify using (3.11) and (3.8) to get (3.14).

- Multiply (3.7) on the right by $a$ and simplify using (3.11) and (3.8) to get (3.15).

- Multiply (3.15) on the left by $c$ and simplify using (3.8) and (3.9) to get (3.16).

- Multiply (3.13) on the right by $d$ and simplify using (3.6) and (3.16) to get (3.17).

- Finally, multiply (3.13) on the left by $a$, multiply (3.15) on the right by $b$, add the results and simplify using (3.3) to get (3.18).

On the other hand, we can derive (3.3)-(3.10) from (3.12)-(3.18) as follows:

- Multiply (3.13) on the left by $a$, add to (3.18) and simplify using (3.15) to get (3.3).

- Multiply (3.13) on the right by $a$ and simplify using (3.14) to get (3.4).

- Multiply (3.15) on the left by $b$ and simplify using (3.14) and (3.17) to get (3.5).

- Multiply (3.13) on the right by $d$ and simplify using (3.16) and (3.17) to get (3.6).

- Multiply (3.13) on the left by $d$, add to (3.18) and simplify using (3.15) to get (3.7).

- The relation (3.8) appears as (3.12).

- Multiply (3.15) on the left by $c$ and simplify using (3.8) and (3.16) to get (3.9).

- Finally, (3.13) and (3.15) imply that $f e=(b+c)(a+d)$; simplify using (3.12), (3.14), (3.16), and (3.17) to get (3.10). 
Therefore, since the two collections of relations (3.3)-(3.10) and (3.12)(3.18) are equivalent, we see that

$$
\begin{aligned}
\bar{C} \simeq \mathbb{Z} / 2\langle a, b, c, d, e, f\rangle /\langle c a=1, b+c+f=0, b a=0, \\
\\
\quad a+d+e=0, c d=0, b d=1, a c+d b=1\rangle .
\end{aligned}
$$

Since $e=a+d$ and $f=b+c$, we can re-write $\bar{C}$ as

$$
\bar{C} \simeq \mathbb{Z} / 2\langle a, b, c, d\rangle /\langle a c+d b=1, b a=0, b d=1, c a=1, c d=0\rangle,
$$

completing the proof of the lemma.

The goal now is to show that $\bar{C}$ is nontrivial, which will imply that $C\left(m\left(10_{161}\right)\right)$ is nontrivial as well.

Lemma 3.2. The algebra

$$
\bar{C}=\mathbb{Z} / 2\langle a, b, c, d\rangle /\langle a c+d b=1, b a=0, b d=1, c a=1, c d=0\rangle
$$

is nontrivial.

Proof. To prove this, we define an action of $\bar{C}$ on $\mathcal{H}$, where $\mathcal{H}$ is a countably infinite-dimensional vector space over $\mathbb{Z} / 2$. Provided we can show this action is nontrivial, this will imply that $\bar{C}$ is nontrivial.

As with any infinite-dimensional vector space, $\mathcal{H}$ can be written as

$$
\mathcal{H}=\mathcal{H}_{1} \oplus \mathcal{H}_{2}
$$

where $\mathcal{H}_{1} \simeq \mathcal{H}_{2} \simeq \mathcal{H}$ as $\mathbb{Z} / 2$-vector spaces, so any map $\mathcal{H} \rightarrow \mathcal{H}_{1} \oplus \mathcal{H}_{2}$ or $\mathcal{H}_{1} \oplus \mathcal{H}_{2} \rightarrow \mathcal{H}$ defines an endomorphism of $\mathcal{H}$.

Fix identifications $\mathcal{H} \cong \mathcal{H}_{1}$ and $\mathcal{H} \cong \mathcal{H}_{2}$ (throughout what follows the symbol $\cong$ will refer to these fixed identifications).

Let $a, b, c, d$ act on $\mathcal{H}$ as follows:

- Define $a: \mathcal{H} \rightarrow \mathcal{H}_{1} \oplus \mathcal{H}_{2}$ by the diagram

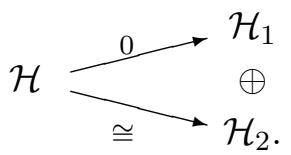

- Define $b: \mathcal{H}_{1} \oplus \mathcal{H}_{2} \rightarrow \mathcal{H}$ by the diagram

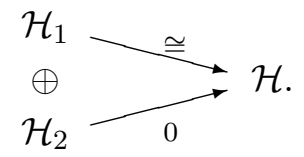

- Define $c: \mathcal{H}_{1} \oplus \mathcal{H}_{2} \rightarrow \mathcal{H}$ by the diagram

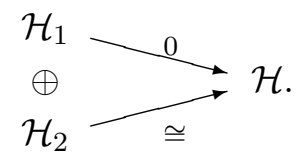


- Define $d: \mathcal{H} \rightarrow \mathcal{H}_{1} \oplus \mathcal{H}_{2}$ by the diagram

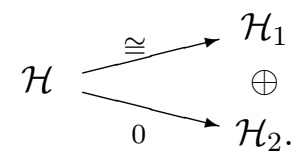

Extending by linearity, the defining relations of $\bar{C}$ are preserved by this action, so the above induces a well-defined action of $\bar{C}$ on $\mathcal{H}$ (alternatively, a representation of $\bar{C}$ into $\operatorname{End}(\mathcal{H}))$. Since the actions of $a, b, c$, and $d$ are clearly nontrivial, this is a nontrivial action, completing the proof of the lemma.

Since $\bar{C}$ is a quotient of $C\left(m\left(10_{161}\right)\right)$, Lemma 3.2 implies that $C\left(m\left(10_{161}\right)\right)$ is nontrivial, completing the proof of Theorem 1.1.

\section{The contact homology of the $m\left(1_{139}\right)$}

Our goal in this section is to prove Proposition 1.1 by showing that 1 is in the image of the differential of Chongchitmate and Ng's conjecturally nondestabilizable $m\left(10_{139}\right)$. This Legendrian $m\left(10_{139}\right)$ is one of two examples given by Chongchitmate and $\mathrm{Ng}$ with nonmaximal Thurston-Bennequin invariants which computations suggest sit atop their own peaks in the $t b-r$ mountain range. That the other - a Legendrian $m\left(12 n_{242}\right)$ - also has vanishing contact homology and characteristic algebra follows from a similar argument to the one given below.

The plat diagram for the $m\left(10_{139}\right)$ given in Figure 2 is obtained from Chongchitmate and Ng's presentation.

The braid word for the plat diagram in Figure 2 is

$6,7,8,9,10,11,12,13,13,5,7,9,11,2,4,6,8,10,11$, $13,12,10,11,9,10,8,9,7,8,6,7,5,6,4,5,3,4,2$

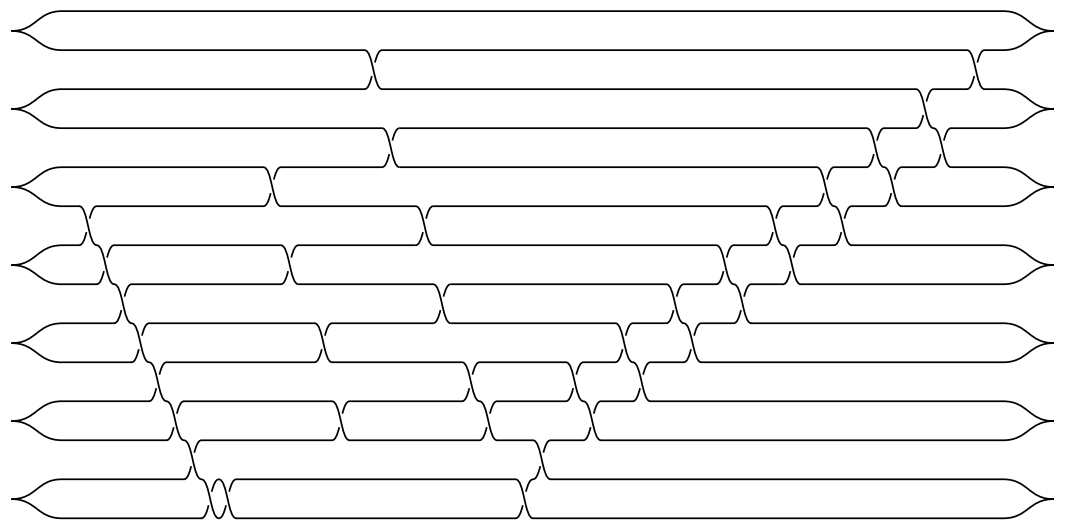

Figure 2. Chongchitmate and Ng's conjecturally nondestabilizable $m\left(10_{139}\right)$. 
In order to prove that the contact homology and characteristic algebra of the $m\left(10_{139}\right)$ are trivial, it suffices to construct an element $a \in A$ such that $\partial a=1$. From the presentation of the differential given in Appendix B, we see that

$$
\begin{aligned}
1=\partial\left(( x _ { 2 } + x _ { 1 0 } ) \left(\left(\left(x_{41} x_{11}+\right.\right.\right.\right. & \left.\left.\left.x_{14} x_{42}\right) x_{15}+x_{41}-x_{44}\right) x_{22}+x_{24}\right) \\
& \left.+\left(x_{4}+x_{16}\right)\left(x_{15} x_{22}+x_{19}\right)+x_{6}+x_{43}\right) .
\end{aligned}
$$

Therefore, the contact homology and characteristic algebra of Chongchitmate and Ng's $m\left(10_{139}\right)$ both vanish over $\mathbb{Z}\left[t, t^{-1}\right]$.

\section{Appendix A. The differential over $\mathbb{Z} / 2$ for the $m\left(1_{161}\right)$}

$$
\begin{aligned}
\partial x_{1} & =0 \\
\partial x_{2} & =x_{1} \\
\partial x_{3} & =0 \\
\partial x_{4} & =x_{3} x_{1} \\
\partial x_{5} & =x_{3} x_{2}+x_{4} \\
\partial x_{6} & =x_{3} \\
\partial x_{7} & =0 \\
\partial x_{8} & =x_{7} \\
\partial x_{9} & =0 \\
\partial x_{10} & =x_{9} \\
\partial x_{11} & =x_{3} \\
\partial x_{12} & =0 \\
\partial x_{13} & =0 \\
\partial x_{14} & =0 \\
\partial x_{15} & =x_{14} \\
\partial x_{16} & =0 \\
\partial x_{17} & =x_{16} \\
\partial x_{18} & =0 \\
\partial x_{19} & =x_{1}+x_{12} x_{4}+x_{12} x_{11} x_{1} \\
\partial x_{20} & =x_{2} x_{13}+x_{12} x_{5} x_{13}+x_{12} x_{11} x_{2} x_{13}+1+x_{12} x_{6}+x_{12} x_{11}+x_{19} x_{13} \\
\partial x_{21} & =x_{2} x_{14}+x_{12} x_{5} x_{14}+x_{12} x_{11} x_{2} x_{14}+x_{12} x_{7}+x_{19} x_{14} \\
\partial x_{22} & =x_{2} x_{15}+x_{12} x_{5} x_{15}+x_{12} x_{11} x_{2} x_{15}+x_{12} x_{8}+x_{19} x_{15}+x_{21} \\
\partial x_{23} & =x_{2} x_{16}+x_{12} x_{5} x_{16}+x_{12} x_{11} x_{2} x_{16}+x_{12} x_{9}+x_{19} x_{16} \\
\partial x_{24} & =x_{2} x_{17}+x_{12} x_{5} x_{17}+x_{12} x_{11} x_{2} x_{17}+x_{12} x_{10}+x_{19} x_{17}+x_{23} \\
\partial x_{25} & =0 \\
\partial x_{26} & =x_{25} \\
\partial x_{27} & =0 \\
\partial x_{28} & =0 \\
\partial x_{29} & =x_{28} x_{25} \\
&
\end{aligned}
$$




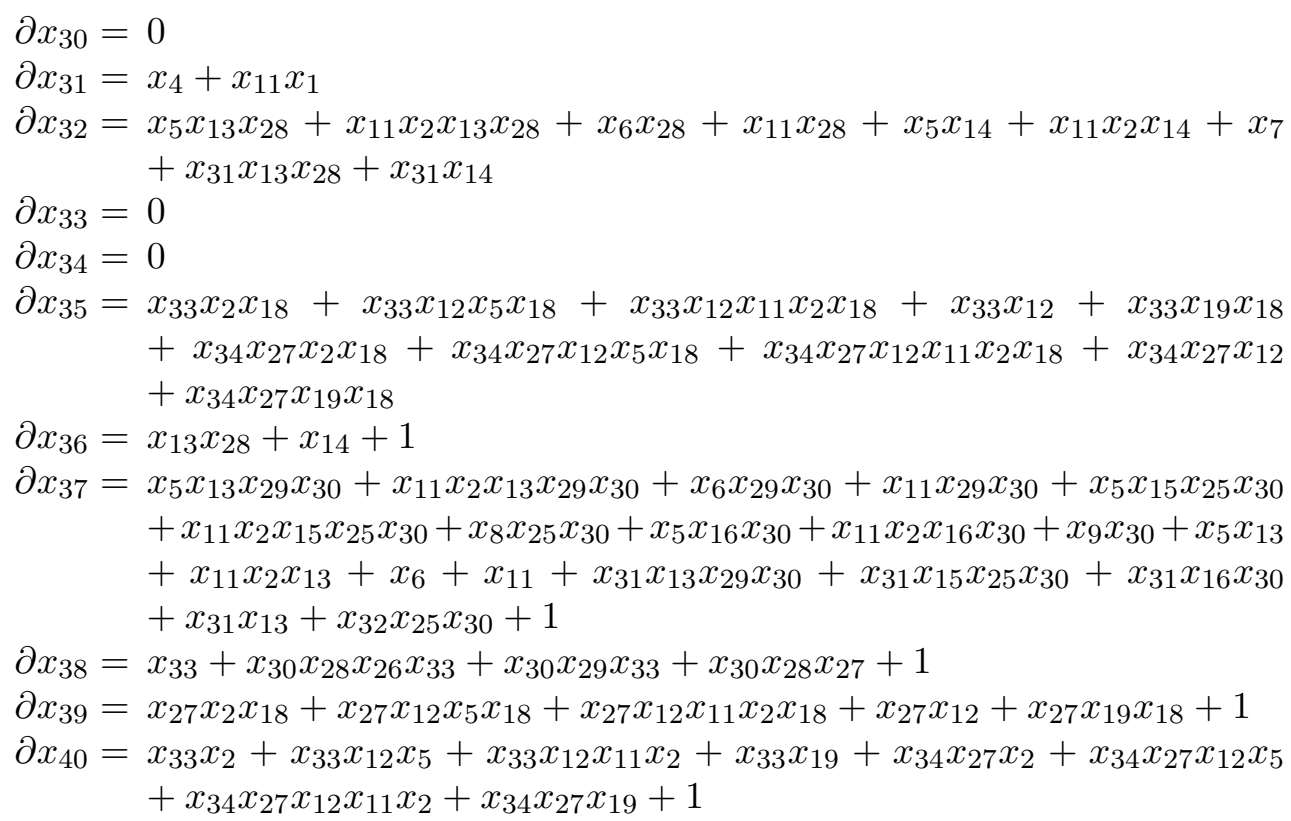

\section{Appendix B. The differential over $\mathbb{Z}\left[t, t^{-1}\right]$ for the $m\left(1_{139}\right)$}

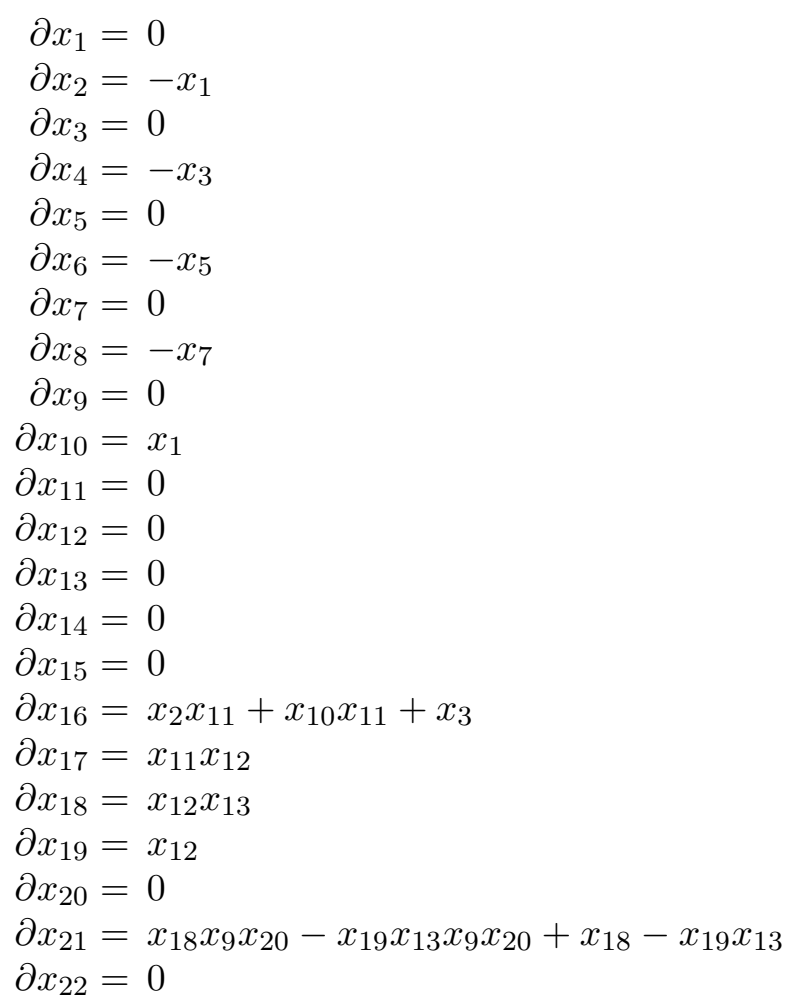




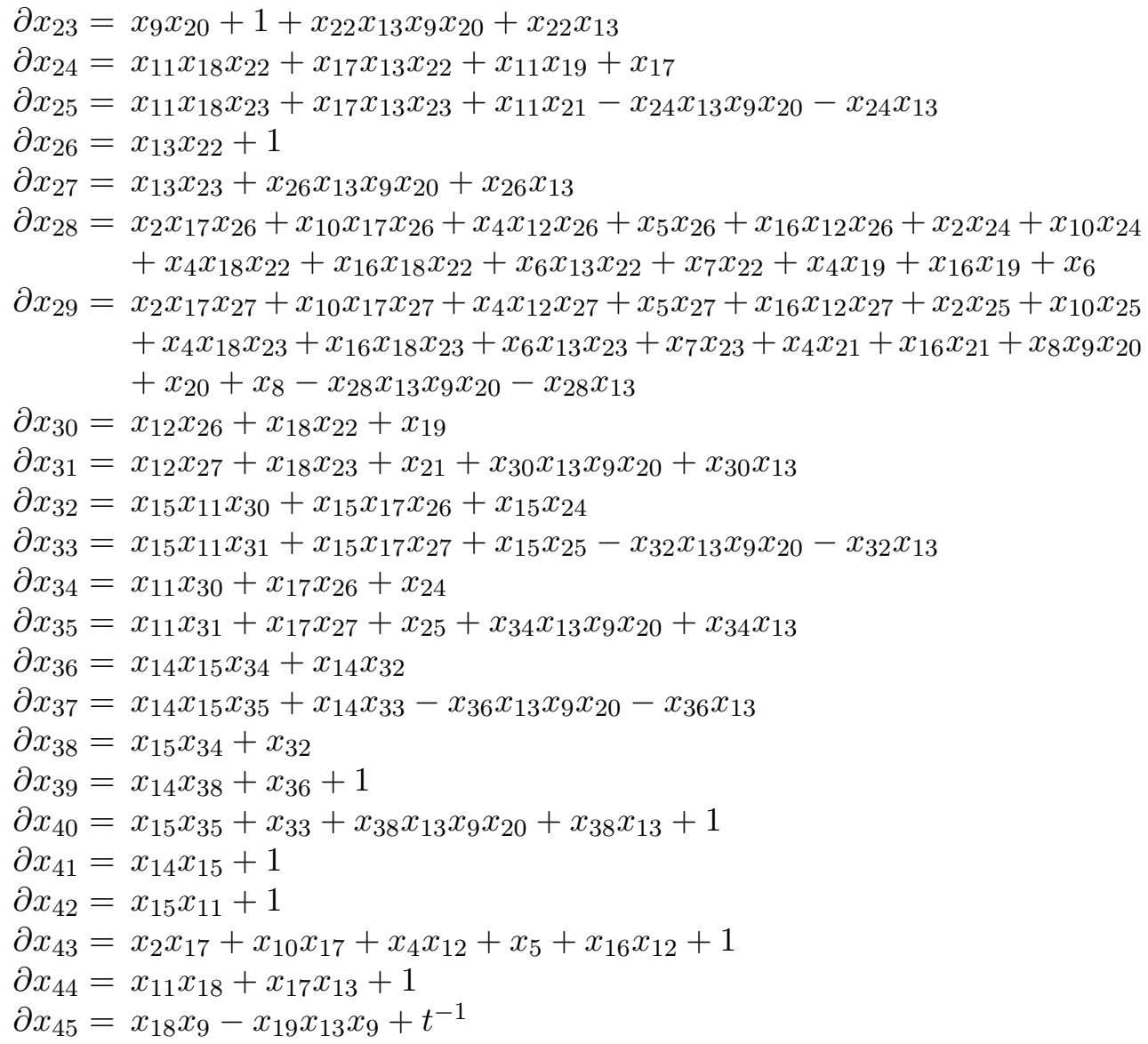

\section{References}

[Che02] Y. Chekanov, Differential algebra of Legendrian links, Invent. Math. 150(3) (2002), 441-483.

[CN09] W. Chongchitmate and L. L. Ng, Legendrian knot atlas, 2009, http://www . math. duke.edu/ ng/atlas/.

[EES05] T. Ekholm, J. B. Etnyre, and M. Sullivan, Orientations in Legendrian contact homology and exact Lagrangian immersions, Internat. J. Math. 16(5) (2005), $453-532$.

[EGH00] Y. Eliashberg, A. Givental and H. Hofer, Introduction to symplectic field theory, Geom. Funct. Anal. Special Volume, Part II (2000), 560-673.

[Eli98] Y. Eliashberg, Invariants in contact topology, in 'Proceedings of the International Congress of Mathematicians', Vol. II (Berlin, 1998), Doc. Math. 1998, Extra Vol. II, 327-338.

[Etn99] J. B. Etnyre, Transversal torus knots, Geom. Topol. 3 (1999), 253-268.

[Etn05] L Legendrian and transversal knots, Handbook of Knot Theory, Elsevier B. V., Amsterdam, 2005, 105-185. 
[EH01] J. B. Etnyre and K. Honda, Knots and contact geometry I: Torus knots and the figure eight knot, J. Symplectic Geom. 1(1) (2001), 63-120.

[EH05] , Cabling and transverse simplicity, Ann. Math. (2) 162(3) (2005), 1305-1333.

[ENS02] J. B. Etnyre, L. L. Ng, and J. M. Sabloff, Invariants of Legendrian knots and coherent orientations, J. Symplectic Geom. 1(2) (2002), 321-367.

[Ng01] L. L. Ng, Invariants of Legendrian links, Ph.D. thesis, Massachusetts Institute of Technology, 2001.

[Ng03]__ Computable Legendrian invariants, Topology 42(1) (2003), 55-82.

Department of Mathematics

Haverford COLleGE

370 Lancaster Ave., Haverford, PA 19041

E-mail address: cshonkwi@haverford.edu

http://www.haverford.edu/math/cshonkwi

Department of Mathematics

Columbia University

MC 4403, 2990 BROADWAY, NEW YORK, NY 10027

E-mail address: shea@math.columbia.edu

http://www.math.columbia.edu/ shea

Received 03/03/2010, accepted 04/09/2010

DSV was partially supported by an NSF Postdoctoral Research Fellowship.

We would like to thank John Etnyre for suggesting that we explore the relationship between the Thurston-Bennequin invariant and Legendrian contact homology. Thanks also to Lenny $\mathrm{Ng}$ and Wutichai Chongchitmate for their work creating the Legendrian knot atlas, Dylan Thurston for his helpful suggestion, and David Fithian for his timesaving Mathematica program. 